\title{
Association between dietary vitamin D intakes and blood pressure in Caucasian and South Asian females: preliminary analysis of the D2-D3 Study
}

\author{
L. Wilson, E. Hall, K. Hart, S. Lanham-New and L. Tripkovic \\ Department of Nutrition and Metabolism, Institute of Biosciences and Medicine, Faculty of Health and Medical Sciences, \\ University of Surrey, Guildford GU2 7XH, UK
}

There is evidence of an inverse association between vitamin D status and blood pressure ${ }^{(1)}$, which indicates a potential role for vitamin D in cardiovascular disease prevention. The work to date has been particularly focused on $25(\mathrm{OH}) \mathrm{D}$ levels ${ }^{(2)}$, but little research has focused on the influence of dietary intake of vitamin D on blood pressure measurements, especially in population groups at risk of vitamin D deficiency. Therefore, the aim of this study was to investigate and compare the association between dietary vitamin D intake and blood pressure in women of both Caucasian $(\mathrm{Cn})$ and South Asian (SA) origin, taking into consideration other dietary influences and anthropometrics.

A total of 88 women $(n 71 \mathrm{Cn}, n 17 \mathrm{SA})$ aged 20-64 years were recruited from the Surrey area as part of the D2-D3 study (a vitamin D food fortification trial). As part of their baseline visit for the D2-D3 study, participants completed a four-day food diary and had anthropometric and blood pressure measurements taken. The four-day food diaries were analysed using DietPlan6 to determine nutrient intakes. Blood pressure was measured in rested participants and the mean of three measurements was taken for systolic blood pressure (SBP), diastolic blood pressure (DBP), mean arterial pressure (MAP), pulse pressure (PP) and heart rate (HR). Analysis was carried out as whole group, between ethnic groups and also between age groups (20-34, 35-49 and 50-64 years) and body mass index (BMI) categories $\left(18-24.9 \mathrm{~kg} / \mathrm{m}^{2}\right.$ and $\left.25-30 \mathrm{~kg} / \mathrm{m}^{2}\right)$.

The overall mean age and BMI $(n 88)$ were $40.3 \pm 13.2$ years and $23.8 \pm 3.5 \mathrm{~kg} / \mathrm{m}^{2}$ respectively. Compared to SA women, the Cn women had a significantly lower body fat percentage (SA: $31.8 \pm 6.6$ vs. C: $28.1 \pm 6.6, p=0.046$ ) and waist-hip ratio (SA: $0.80 \pm 0.07$ vs C: $0.76 \pm 0.07, p=0.02$ ) but there were no differences in age or BMI.

Although dietary intakes were markedly different between ethnic groups (data not presented), vitamin D intakes were not significantly different $(\mathrm{Cn}: 2.7 \pm 1.9 \mu \mathrm{g} / \mathrm{d} v s$. SA: $2.2 \pm 2.7 \mu \mathrm{g} / \mathrm{d})$. The average intake of vitamin D was $2.6 \pm 2.0 \mu \mathrm{g} / \mathrm{d}(105.2 \pm 81.4 \mathrm{IU} / \mathrm{d})$. The results show that dietary vitamin D intake did not correlate with blood pressure measurements in the group as a whole nor in any sub-group analysis taking into account BMI and age.

Further analysis into the blood pressure measurements showed that Caucasian women had a significantly higher PP $(\mathrm{Cn}: 39.1 \pm 8.4$ vs. SA:33.8 $\pm 8.8, p=0.024)$, but there were no significant differences in SBP, DBP, MAP or HR. SBP consistently increased with age, body weight, BMI and waist circumference $(p<0.001)$; as did DBP $(p<0.001)$; and MAP $(p<0.001)$ which was consistent in sub-group age analysis, except in women aged 50-64 years. PP increased only with age $(p<0.001)$ and HR increased only with weight $(p=0.043)$

This works confirms the association between age and BMI and blood pressure ${ }^{(3)(4)}$, but did not find any correlation between dietary vitamin D intakes and blood pressure at the levels seen in this sample group. Although the UK does not have a recommended dietary intake (RDI) for men and women aged 5-64 years, there is an RDI of $10 \mu \mathrm{g} / \mathrm{d}$ (400 IU/d) set for those who are at risk of deficiency. As the average intake of vitamin D in this study was far below what is considered adequate to support essential metabolic pathways, it would be valid and interesting to investigate whether a higher and sustained level of vitamin D intake can positively influence blood pressure with the potential consequence of ameliorating CVD risk.

1. Burgaz et al. (2011) J of Hypertension 29, 636-645.

2. Pittas et al. (2010) Ann Intern Med 152, 307-314.

3. Drøyvold et al. (2005) Int J Obes 29(6), 650-655.

4. Kannel WB (1996) JAMA 275(20), 1571-1576. 